\title{
Impact of Brief Smoking Cessation Intervention on Abstinence Rate and Glycaemic Control in Patients with Diabetes Mellitus: A Randomised Controlled Trial
}

Khansaa Albaroodi ( $\sim$ khansaa.albaroodi@hotmail.com )

Alzahrawy University college

Syed Azhar Syed Sulaiman

Universiti Sains Malaysia

Ahmed Awaisu

Qatar University

Asrul Shafie

Universiti Sains Malaysia

\section{Research Article}

Keywords: diabetes, smoking cessation intervention, glycaemic control, blood pressure, quitting rate

Posted Date: January 28th, 2021

DOI: https://doi.org/10.21203/rs.3.rs-149819/v1

License: (c) (i) This work is licensed under a Creative Commons Attribution 4.0 International License. Read Full License 


\section{Abstract}

Aims: This study aimed to evaluate the impact of brief smoking cessation intervention on smoking cessation outcomes as well as on glycaemic and blood pressure control among patients with diabetes.

Methods: This was a randomised controlled trial involving patients with diabetes who smoked tobacco and attended the out-patient Diabetes Clinic at Penang Hospital in Malaysia. One hundred forty participants were randomised into either control $(n=70)$ or intervention $(n=70)$ groups. The intervention consisted of a 5-minute physician-delivered brief counselling on tobacco cessation using 5A's strategy (Ask, Advise, Assess, Assist, and Arrange) in addition to usual care for patients with diabetes, while the control group received only the usual care.

Results: There was no significant difference between the two groups with respect to glucose control, blood pressure levels, and smoking abstinence rates $(P>0.05)$. Furthermore, significant main effects were found between the groups with respect to the number of cigarettes smoked per day $(F[1,116]=6.306)$.

Conclusions: Brief smoking cessation intervention did not result in better abstinence rates or glycaemic control in patients with diabetes. However, it resulted in reduction in the number of cigarettes smoked per day over the study period (6 months), which is the first step in the tobacco cessation process.

\section{Trial registration}

Approval for the conduct of this study was granted by the Medical Research Ethics Committee of the Ministry of Health, Malaysia and the Clinical Research Centre at Hospital Pulau Pinang, Malaysia (NMRR11-477-9538) at (05-10-2011).

\section{Background}

Smoking tobacco is considered as an avoidable cause of morbidity and premature mortality worldwide (1). In recent years, there has been an alarming increase in the prevalence of diabetes mellitus worldwide as well as in Malaysia (2). The association between tobacco smoking and diabetes is becoming increasingly important. Several studies have reported the association between tobacco smoking and poor glycaemic control and that smoking increases morbidity and mortality among patients with both type 1 and 2 diabetes (3-8). Furthermore, smokers had twice the risk for developing diabetes than non-smokers (9). A meta-analysis and systematic review identified an association between active tobacco smoking and diabetes mellitus (10). Quitting tobacco reduces mortality risks among patients with diabetes within several years after quitting (11). Therefore, tobacco cessation is strongly recommended to improve glycaemic control and to slow the development of diabetic complications $(1,12)$. The International Diabetes Federation guidelines for type 2 diabetes included tobacco cessation advice as a standard of care to reduce or stop tobacco consumption $(2,13)$. This intervention is anticipated to improve clinical outcomes of diabetes care and ultimately quality of life. Despite the importance of improving glycaemic control and screening for microvascular complications, it may be even more important to detect major macrovascular risk factors and control them. 
Previous studies have investigated the effectiveness of tobacco cessation interventions among patients with diabetes who smoke $(9,14-21)$. The major limitations of these studies included, but are not limited to, some are not RCT some small sampling, some inconclusive results. Moreover, most studies focused on evaluating the effectiveness of the interventions on smoking cessation outcomes such as smoking abstinence rate, and number of cigarettes smoked per day, but rarely investigated the impact of the interventions on diabetes control. Consequently, rigorous studies are needed to evaluate the effect of structured tobacco cessation interventions tailored to patients with diabetes on both smoking and diabetes-related outcomes. Previous tobacco cessation intervention studies in other disease conditions such as tuberculosis (TB) (22-25) and chronic obstructive pulmonary disease (COPD) have shown the benefits of brief counselling delivered by healthcare professionals on smoking and disease-related outcomes $(26,27)$. This study aimed to evaluate the impact of a physician-delivered disease-specific brief tobacco cessation intervention in diabetes care on quitting rate, the number of cigarette smoked per day, glycaemic control, blood pressure (BP), and lipid profile. The individually-tailored intervention aimed at helping patients with diabetes who smoke to quit smoking.

\section{Methods}

\section{Study design and participants}

A randomized controlled trial was carried out and involved patients with diabetes who smoked tobacco and who attended the out-patient Diabetes Clinic at Penang Hospital in Malaysia. Randomly participants were assigned into one of the two study groups: control group who received routine diabetes care counselling or intervention group who received diabetes-specific brief tobacco cessation counselling in addition to the routine diabetes care counselling. Figure 1 illustrates the study design. The study was conducted between March 2012 and August 2013. Each patient was followed for three sequential visits. The medical records of the patients included in the study were prospectively reviewed for the laboratory data.

Patients were included into the study if they: (1) have a documented diagnosis of type 1 or type 2 diabetes mellitus; (2) were currently smoking tobacco (have smoked at least 100 cigarettes during lifetime and have smoked within the last month) and; (3) could speak English and/or Malay.

\section{Sample size and sampling method}

The sample size needed for the RCT study was calculated using the equation below (28):

$$
m=C \times \frac{\pi_{1}\left(1-\pi_{1}\right)+\pi_{2}\left(1-\pi_{2}\right)}{\left(\pi_{1}-\pi_{2}\right)^{2}}
$$

where $\mathrm{C}=7.9$ for $80 \%$ power, $\pi_{1}$ and $\pi_{2}$ are the proportions of the primary outcome measure in the intervention and control groups which were estimated from the literature (14). The minimum estimated 
sample size for each group was 48 patients. The sample size per group was increased by $30 \%$, resulting in 67 patients. This study enrolled 70 eligible patients per group to compensate for patients lost to followup and non-response.

\section{Randomisation}

Participants were randomly assigned to intervention or control groups using a computer-generated allocation method.

\section{Description of the brief tobacco cessation intervention program for patients with diabetes}

The tobacco cessation protocol consisted of performing a routine assessment (asking) for tobacco use; providing advice on the importance of quitting; performing an assessment of the tobacco user's readiness to quit; assisting in setting a quit date; and making arrangements for follow-up visits (29). This protocol is popularly known as the $5 \mathrm{~A}^{\prime}$ s strategy. The intervention in this study was delivered by physicians (who provided diabetes care to the participants) and nurses who assisted during follow-up and monitoring. The physicians were specially trained on providing tobacco cessation intervention and were supported by a booklet that provided specific information on counselling, which was adapted from tobacco cessation guidelines. Patients were counselled during each routine visit to the endocrine clinic (which should be every 3 to 4 months) $(29,30)$.

\section{Outcome measures}

The primary outcome that was measured in every visit (every 3 to 4 months) included mean glycated haemoglobin ( $\mathrm{HbA} 1 \mathrm{c})$, abstinence rates, and the mean number of cigarette smoked per day. The secondary outcome measures included blood pressure and lipid profile. Smoking cessation was validated using breath carbon monoxide (CO) test as a biochemical measure. A breath carbon monoxide (CO) monitor called Smokerlyzer piCO + was used to verify smoking cessation (31).

\section{Data analyses}

The collected data were analysed using SPSS (version 18.0) software package (SPSS Inc., Chicago, IL). Descriptive and inferential statistics were used as appropriate. To determine the differences between the intervention and the control groups with respect to outcome measures ( $\mathrm{HbA1}$ c, number of cigarette smoked per day, BP, lipid profile, CO level), the independent t-test, Mann-Whitney U test and Pearson's chisquare test were used as appropriate. To determine the differences between the control and intervention groups in repeated time points, a mixed ANOVA test was applied $(32,33)$. P-value $\leq 0.05$ was considered statistically significant.

\section{Results}

A total of 140 patients with diabetes who smoke tobacco were initially enrolled in the study. However, 14 were lost to follow-up due to several reasons: seven patients relocated from Pulau Pinang, two died, three withdrew from participating, and two were excluded due to incomplete data. Thus, 126 participants (X 
number of participants in the intervention group and $Y$ number of participants in the control group) were

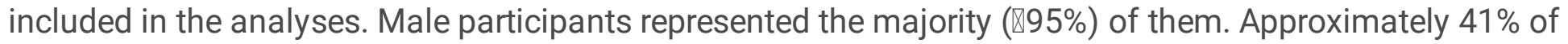
the participants were Malay and the rest of them were Chinese and Indian.

Table 1 demonstrates that a non-significant difference between patients in the two groups was observed with respect to their glucose control ( $\mathrm{HbA1c}$ ) and the BP levels (systolic and diastolic BP). In general, a high mean $\mathrm{HbA} 1 \mathrm{c}$ level was observed in the two groups over the three visits based on the Malaysian Clinical Practice Guideline for normal values reference (7-9\% fair control) (34). However, the BP of the patients in both groups was well controlled throughout the study period $(<130 / 80 \mathrm{mmHg})$ and was within the normal range of BP as determined by the Malaysian Clinical Practice Guidelines 2004 for normal values reference (34) (Table 1). However, a significant difference was observed in the number of cigarettes smoked per day between the two groups during the first two visits (intervention was better than the control group) (Table 1), but this difference was not significant in their last visit.

Table 1 presents the mean level of $\mathrm{CO}$ as ppm and the \% $\mathrm{CO} \mathrm{Hb}$, which stratifies the study participants as low-frequency smokers or smokers according to the manufacturer's (Bedfont) guide. No significant differences were noted between patients in the two groups during the first two visits with respect to $\mathrm{CO}$ level. During the third visit, the control group had significantly lower $\mathrm{CO}$ concentration compared with the intervention group $(9.2 \pm 6 \mathrm{ppm}$ vs. $11.8 \pm 6.9 \mathrm{ppm} ; \mathrm{p}=0.032)$. Quitting rate is defined as the proportion of patients who achieved success in quitting smoking tobacco at their second and/or last visit. There was no significant difference between the two groups in terms of abstinence rate at 6 months. However, the proportion of patients who reduced their cigarette consumption in both groups was considered favourable. Table 1 demonstrates a non-significant difference between patients in the two groups with respect to their lipid profile (TG, $L D L, H D L$, and total cholesterol). Among the intervention group the number of cigarettes smoked per day, systolic BP and diastolic BP revealed a significant difference between baseline and the last visit. 
Table 1

Outcome measures for tobacco cessation intervention among patients with diabetes

$\begin{array}{ll}\text { Item } & \text { Intervention group }\left(n=\quad \begin{array}{l}\text { Control group }(n=63) \text { Mean } \pm \quad P \text { Value } \\ \text { SD }\end{array}\right. \\ & \text { Mean } \pm \text { SD }\end{array}$

HbA1c (\%)

\begin{tabular}{llll} 
First visit (baseline) & $8.6 \pm 2.6$ & $8.8 \pm 2.5$ & $0.427^{\star \star}$ \\
\hline Second visit & $8.4 \pm 2$ & $8.6 \pm 2.3$ & $0.819 \star *$ \\
\hline Third visit & $8.8 \pm 2.5$ & $8.7 \pm 2.4$ & $0.762^{\star}$
\end{tabular}

\section{Systolic BP (mmHg)}

\begin{tabular}{llll} 
First visit (baseline) & $126.4 \pm 15.3$ & $127.9 \pm 29$ & $0.270^{\star *}$ \\
\hline Second visit & $123.6 \pm 16.1$ & $124.5 \pm 25.8$ & $0.641^{\star *}$ \\
\hline Third visit & $120.8 \pm 23.1$ & $124.3 \pm 24.4$ & $0.304^{\star *}$
\end{tabular}

\section{Diastolic BP ( $\mathrm{mmHg})$}

\begin{tabular}{llll} 
First visit (baseline) & $75.6 \pm 8.1$ & $76.1 \pm 13.2$ & $0.809^{*}$ \\
\hline Second visit & $73.5 \pm 13.3$ & $73.9 \pm 14.1$ & $0.866^{*}$ \\
\hline Third visit & $71.1 \pm 8.5$ & $72.2 \pm 15.4$ & $0.152^{\star *}$
\end{tabular}

\section{No. of cigarettes/day}

\begin{tabular}{llll}
\hline First visit (baseline) & $16.51 \pm 9.7$ & $12.81 \pm 9.2$ & $0.009(\mathrm{~S})^{\star *}$ \\
\hline Second visit & $12.97 \pm 9.3$ & $9.05 \pm 7.4$ & $0.005(\mathrm{~S})^{\star *}$ \\
\hline Third visit & $11.03 \pm 8.8$ & $8.83 \pm 7.4$ & 0.120 **
\end{tabular}

\section{Co level, ppm}

\begin{tabular}{llll} 
First visit (baseline) & $12.8 \pm 9.7$ & $11.1 \pm 7.7$ & $0.271^{\star}$ \\
\hline Second visit & $10.5 \pm 5.8$ & $11.8 \pm 11.5$ & $0.477^{\star \star}$ \\
\hline Third visit & $11.8 \pm 6.9$ & $9.2 \pm 6$ & $0.032(\mathrm{~S})$ \\
\hline CO level, Hb & & & \\
\hline First visit (baseline) & $2.6 \pm 1.6$ & $2.4 \pm 1.2$ & $0.282^{\star}$ \\
\hline Second visit & $2.3 \pm 0.9$ & $2.5 \pm 1.8$ & $0.426^{\star \star}$
\end{tabular}

$\mathrm{CO}$ ppm: carbon monoxide level in parts per million; $\mathrm{CO} \mathrm{Hb}$ : carbon monoxide level in the haemoglobin;

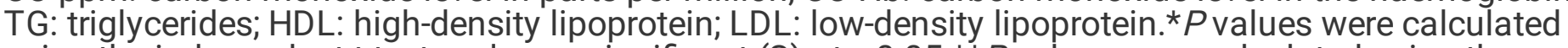
using the independent $t$-test and were significant $(S)$ at $<0.05 * \star *$ values were calculated using the Mann-Whitney U test and were significant $(S)$ at $<0.05 . \star \star \star P$ values were calculated using Pearson's chisquare test and were significant (S) at $<0.05$. 


\begin{tabular}{|c|c|c|c|}
\hline \multirow[t]{2}{*}{ Item } & $\begin{array}{l}\text { Intervention group }(n= \\
63)\end{array}$ & $\begin{array}{l}\text { Control group }(n=63) \text { Mean } \pm \\
\text { SD }\end{array}$ & $P$ Value \\
\hline & \multicolumn{3}{|l|}{ Mean \pm SD } \\
\hline Third visit & $2.5 \pm 1.1$ & $2.1 \pm 0.9$ & $0.038(\mathrm{~S})^{\star}$ \\
\hline \multicolumn{3}{|l|}{ Abstinence rate } & $0.934 * \star \star$ \\
\hline No change & $31(49.2)$ & $33(52.4)$ & \\
\hline Reduced & $28(44.4)$ & $26(41.3)$ & \\
\hline Quit & $4(6.3)$ & $4(6.3)$ & \\
\hline \multicolumn{4}{|l|}{ TG (mmol/L) } \\
\hline At baseline & $2 \pm 1.2$ & $2.4 \pm 3.4$ & $0.631^{\star \star}$ \\
\hline Third visit & $2 \pm 1$ & $2.2 \pm 1.8$ & $0.631^{\star \star}$ \\
\hline \multicolumn{4}{|l|}{ HDL (mmol/L) } \\
\hline At baseline & $1.1 \pm 0.3$ & $1.1 \pm 0.3$ & $0.398 * \star$ \\
\hline Third visit & $1.1 \pm 0.3$ & $1.1 \pm 0.2$ & $0.944^{\star \star}$ \\
\hline \multicolumn{4}{|l|}{ LDL (mmol/L) } \\
\hline At baseline & $2.8 \pm 0.9$ & $3.1 \pm 1.1$ & 0.344 \\
\hline Third visit & $2.7 \pm 0.7$ & $2.8 \pm 1.3$ & 0.605 \\
\hline \multicolumn{4}{|l|}{$\begin{array}{l}\text { Total cholesterol } \\
\text { (mmol/L) }\end{array}$} \\
\hline At baseline & $4.79 \pm 0.9$ & $5.1 \pm 1.6$ & $0.572^{\star \star}$ \\
\hline Third visit & $4.71 \pm 0.9$ & $4.8 \pm 1.4$ & $0.661^{\star *}$ \\
\hline \multicolumn{4}{|c|}{ 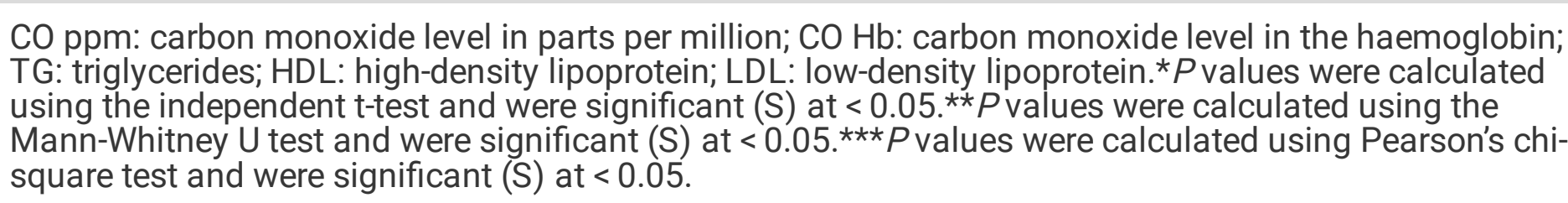 } \\
\hline
\end{tabular}

Table 2 illustrates the repeated measures effects expressed as $F$ values: number of cigarettes smoked per day $F(1.662,192.768)=41.736$, systolic blood pressure $F(2,212)=4.899$, and diastolic blood pressure $F$ $(2,212)=9.108$. 
Table 2

Differences in the study outcome measures pre- and post- tobacco cessation intervention

\begin{tabular}{|c|c|c|c|c|c|}
\hline Source Measure & & Df & $\mathbf{F}$ & Sig. & $\begin{array}{l}\text { Partial Eta- } \\
\text { Squared }\end{array}$ \\
\hline HbA1c & Huynh-Feldt & 1.914 & 1.791 & 0.171 & 0.019 \\
\hline HbA1c*patient group & Huynh-Feldt & 1.914 & 0.569 & 0.560 & 0.006 \\
\hline Error & Huynh-Feldt & 176.104 & & & \\
\hline Systolic BP & $\begin{array}{l}\text { Sphericity } \\
\text { Assumed }\end{array}$ & 2 & 4.899 & 0.008 & 0.044 \\
\hline Systolic BP*patient group & $\begin{array}{l}\text { Sphericity } \\
\text { Assumed }\end{array}$ & 2 & 0.253 & 0.776 & 0.002 \\
\hline Error & $\begin{array}{l}\text { Sphericity } \\
\text { Assumed }\end{array}$ & 212 & & & \\
\hline Diastolic BP & $\begin{array}{l}\text { Sphericity } \\
\text { Assumed }\end{array}$ & 2 & 9.108 & $<.001$ & 0.079 \\
\hline Diastolic BP*patient group & $\begin{array}{l}\text { Sphericity } \\
\text { Assumed }\end{array}$ & 2 & 0.350 & 0.705 & 0.003 \\
\hline Error & $\begin{array}{l}\text { Sphericity } \\
\text { Assumed }\end{array}$ & 212 & & & \\
\hline No. of cigarettes/day & Huynh-Feldt & 1.662 & 41.736 & $<.001$ & 0.265 \\
\hline $\begin{array}{l}\text { No. of cigarettes/day* patient } \\
\text { group }\end{array}$ & Huynh-Feldt & 1.662 & 1.038 & 0.345 & 0.009 \\
\hline Error & Huynh-Feldt & 192.768 & & & \\
\hline CO, ppm & $\begin{array}{l}\text { Sphericity } \\
\text { Assumed }\end{array}$ & 2 & 1.448 & 0.237 & 0.013 \\
\hline CO, ppm, *patient group & $\begin{array}{l}\text { Sphericity } \\
\text { Assumed }\end{array}$ & 2 & 3.021 & 0.051 & 0.027 \\
\hline Error & $\begin{array}{l}\text { Sphericity } \\
\text { Assumed }\end{array}$ & 220 & & & \\
\hline TG & $\begin{array}{l}\text { Sphericity } \\
\text { Assumed }\end{array}$ & 1 & 0.012 & 0.914 & 0.00 \\
\hline TG*atient group & $\begin{array}{l}\text { Sphericity } \\
\text { Assumed }\end{array}$ & 1 & 0.543 & 0.463 & 0.006 \\
\hline
\end{tabular}

The $P$ value is significant (S) at $<0.05$; BMI: body mass index; No. of cigarettes/day: number of cigarettes per day; HbA1c: glycated haemoglobin; Systolic BP: systolic blood pressure; Diastolic BP: diastolic blood pressure; LDL: low-density lipoprotein; HDL: high-density lipoprotein; TG: triglycerides; CO ppm: carbon monoxide level in parts per million; $\mathrm{CO} \mathrm{Hb}$ : carbon monoxide level in the haemoglobin 


\begin{tabular}{|c|c|c|c|c|c|}
\hline \multicolumn{2}{|l|}{ Source Measure } & \multirow{2}{*}{$\begin{array}{l}\text { Df } \\
86\end{array}$} & \multirow[t]{2}{*}{$\mathbf{F}$} & \multirow[t]{2}{*}{ Sig. } & \multirow{2}{*}{$\begin{array}{l}\text { Partial Eta- } \\
\text { Squared }\end{array}$} \\
\hline Error & $\begin{array}{l}\text { Sphericity } \\
\text { Assumed }\end{array}$ & & & & \\
\hline HDL & $\begin{array}{l}\text { Sphericity } \\
\text { Assumed }\end{array}$ & 1 & 1.307 & 0.256 & 0.015 \\
\hline HDL*patient group & $\begin{array}{l}\text { Sphericity } \\
\text { Assumed }\end{array}$ & 1 & 0.670 & 0.415 & 0.008 \\
\hline Error & $\begin{array}{l}\text { Sphericity } \\
\text { Assumed }\end{array}$ & 84 & & & \\
\hline LDL & $\begin{array}{l}\text { Sphericity } \\
\text { assumed }\end{array}$ & 1 & 1.077 & 0.302 & 0.013 \\
\hline LDL*patient group & $\begin{array}{l}\text { Sphericity } \\
\text { Assumed }\end{array}$ & 1 & 0.233 & 0.630 & 0.003 \\
\hline Error & $\begin{array}{l}\text { Sphericity } \\
\text { Assumed }\end{array}$ & 81 & & & \\
\hline Total Cholesterol & $\begin{array}{l}\text { Sphericity } \\
\text { Assumed }\end{array}$ & 1 & 1.300 & 0.257 & 0.015 \\
\hline Cholesterol*patient group & $\begin{array}{l}\text { Sphericity } \\
\text { Assumed }\end{array}$ & 1 & 0.528 & 0.469 & 0.006 \\
\hline Error & $\begin{array}{l}\text { Sphericity } \\
\text { Assumed }\end{array}$ & 87 & & & \\
\hline \multicolumn{6}{|c|}{$\begin{array}{l}\text { The } P \text { value is significant }(\mathrm{S}) \text { at }<0.05 \text {; BMI: body mass index; No. of cigarettes/day: number of } \\
\text { cigarettes per day; HbA1c: glycated haemoglobin; Systolic BP: systolic blood pressure; Diastolic BP: } \\
\text { diastolic blood pressure; LDL: low-density lipoprotein; HDL: high-density lipoprotein; TG: triglycerides; } \\
\text { CO ppm: carbon monoxide level in parts per million; CO Hb: carbon monoxide level in the haemoglobin }\end{array}$} \\
\hline
\end{tabular}

Significant main effects were found among participants in the different groups with respect to the number of cigarettes smoked per day (Table 3) $\mathrm{F}(1,116)=6.306)$. 
Table 3

Differences between control and intervention groups in terms of the study outcome

\begin{tabular}{|c|c|c|c|c|}
\hline Source & Df & $\mathbf{F}$ & Sig. & Partial Eta-Squared \\
\hline No. of cigarettes/day (patient group) & 1 & 6.306 & 0.013 & 0.052 \\
\hline Error & 116 & & & \\
\hline HbA1c (patient group) & 1 & 0.033 & 0.855 & 0.000 \\
\hline Error & 92 & & & \\
\hline Systolic BP (patient group) & 1 & 0.272 & 0.603 & 0.003 \\
\hline Error & 106 & & & \\
\hline Diastolic BP (patient group) & 1 & 0.429 & 0.514 & 0.004 \\
\hline Error & 106 & & & \\
\hline CO, ppm (patient group) & 1 & 0.792 & 0.376 & 0.007 \\
\hline Error & 110 & & & \\
\hline $\mathrm{CO}, \mathrm{Hb}$ (patient group) & 1 & 0.589 & 0.445 & 0.005 \\
\hline Error & 109 & & & \\
\hline TG (patient group) & 1 & 0.032 & 0.859 & 0.000 \\
\hline Error & 86 & & & \\
\hline LDL (patient group) & 1 & 1.037 & 0.312 & 0.013 \\
\hline Error & 81 & & & \\
\hline HDL (patient group) & 1 & 0.056 & 0.813 & 0.001 \\
\hline Error & 84 & & & \\
\hline Total Cholesterol (patient group) & 1 & 0.611 & 0.437 & 0.007 \\
\hline Error & 87 & & & \\
\hline \multicolumn{5}{|c|}{$\begin{array}{l}\text { The } P \text { value is significant }(\mathrm{S}) \text { at }<0.05 \text {; BMI: body mass index; No. of cigarettes/day: number of } \\
\text { cigarettes per day; } \mathrm{HbA1c} \text { glycated haemoglobin; Systolic BP: systolic blood pressure; Diastolic BP: } \\
\text { diastolic blood pressure; } \mathrm{LDL} \text { : low-density lipoprotein; HDL: high-density lipoprotein; TG: triglycerides; } \\
\text { CO ppm: carbon monoxide level in parts per million; CO Hb: carbon monoxide level in the haemoglobin. }\end{array}$} \\
\hline
\end{tabular}

\section{Discussion}

Study results revealed a significant effect of the intervention throughout the study period among all participants with respect to the number of cigarettes smoked per day, however this effect was not significant but obvious in terms of blood pressure. Furthermore, differences between participants in the control and intervention groups in terms of the cigarettes smoked per day can be considered one of the 
main findings of this stud. Despite randomisation of subjects into the groups, patients in the control group smoked less number of cigarettes and had lower $\mathrm{CO}$ level than those in the intervention group (Table 1). However, patients in the intervention group demonstrated improvements in these terms. Furthermore, we observed a clear decrease in the mean number of cigarettes smoked per day between the baseline and third visits as well as a significant difference between the two groups for first and second visits, and these results were confirmed by the multivariate longitudinal analyses. These results can direct future research in this setting. The decrease in the number of cigarettes smoked can enhance future quitting attempts and the success rate (35). Previous studies have reported conflicting results. For instance, a study conducted in Indonesia had reported a non-significant difference between the patient groups with respect to the number of cigarettes smoked (18). However, a study by Canga et al. (14) had demonstrated similar results with the current study in which the mean number of cigarettes between both groups showed significant differences in favour of the intervention group after 6 months (14).

We assume that smoking cessation is a major determinant of glycaemic control, in addition to other measures for diabetes management. Participants' glucose control did not differ over the study period within or between the groups. It is possible that the duration of the study follow-up was insufficient to reveal a clear difference in glycaemic control. In addition, this study relies on a brief cognitive behavioural therapy (CBT) only rather than combination of CBT and pharmacotherapy which has been shown to give better abstinence rate. Therefore, the present study utilized low-intensity intervention strategy witho only 5minute brief smoking cessation advice delivered by the physician. However, a study by Hokanson and colleagues in 2006 revealed an improvement in the HbA1c level over the study period that may have been related to the long study period of approximately three years (17). Another study in France reported a significant decrease in the glycated haemoglobin level (16). Furthermore, participants' BP significantly improved over the study period, as confirmed by the repeated measures effects. As BP can exhibit changes faster than any other measure and many studies showed that sudden quitting can lead to an increment in the BP rather than decrement (36-39), however this study participants reduced the number of cigarette smoked rather than cut it down that aligned with BP improvement (40). The lack of differences between the two groups may relate to random patients' selection and not according to their willingness to stop tobacco smoking based on the transtheoretical model's stage of change. The Hokanson study yielded different results, as there were no differences in BP between the participants over the study period (17).

One limitation of the present study was the use of Smokerlyzer piCO + to measure the CO level for verification of tobacco cessation. This tool cannot be considered as accurate as urine cotinine, because patients can inhale environmental $\mathrm{CO}$ from other sources that may tend to give false-positive results. The patients' CO levels decreased over the study period, consistent with the decrease in the number of cigarettes smoked; however, these results were not significant based on inferential analyses. The higher $\mathrm{CO}$ level in the intervention group compared with the control group comes consistent with the higher number of cigarettes smoked by them. A significant proportion of patients exhibited a reduction in the number of cigarettes smoked, but the number of patients who succeeded in quitting was small. Furthermore, there was no difference in the quitting rate between the two groups, which may be the result of the short study period (6 months only), absence of NRT to boost the intervention effect and random selection of patients

Page $11 / 18$ 
in the two groups not according to their willingness to quit smoking. A randomised controlled trial in Spain reported opposite findings in the difference in the quitting rate between the two groups; the rate of quitting cessation was 7.5-fold higher in the intervention group than the control group (14). Another randomised controlled trial in Canada showed no differences between the intervention and control groups after 6 months, although there was a difference at the 3-month time point (17).

No significant differences were observed neither between patients in the two groups with respect to their lipid profile (TG, and LDL, HDL, and total cholesterol) and nor in their repeated measures over the study period. Although patients in the two groups exhibited improved total cholesterol after the study, the improvement was insignificant. To improve and identify differences in patients' lipid profiles, long-term studies would be required.

\section{Conclusion}

In conclusion, reduction in the number of cigarettes smoked per day over the study period is the first step in the tobacco cessation process. This study finding are the cornerstone for future studies in tobacco cessation interventions among patients with diabetes in Malaysia; future research with more intensive counselling and adjunct nicotine replacement therapy with it as well as following the participants for longer period is highly recommended.

\section{Abbreviations}

BP: blood pressure

CO: carbon monoxide

LDL: low density lipoprotein

HDL: high density lipoprotien

TG: triglycerides

$\mathrm{RCT}$ : randomised controlled trial

COPD: chronic obstructive pulmonary disease

5A's strategy: ask, advise, assess, assist, and arrange

HB: haemoglobin

HbA1c: glycated haemoglobin

\section{Declarations}




\section{Ethics approval and consent to participate}

Approval for the conduct of this study was granted by the Medical Research Ethics Committee of the Ministry of Health, Malaysia and the Clinical Research Centre at Hospital Pulau Pinang, Malaysia. All participants were agreed to participate and signed an informed consent form upon participation in the study. All methods were carried out in accordance with relevant guidelines and regulations.

\section{Consent for publication}

Not applicable.

\section{Availability of data and materials}

All data generated during this study are included in this article and its supplementary information files.

\section{Competing interests}

The authors declare that they have no competing interests.

\section{Funding}

There was no financial support for this research.

\section{Authors' contributions}

Albaroodi K.A.I. set the study design, data collection, data analyses, reporting results and preparing the manuscript, Syed Sulaiman S.A. supervised the whole work, Awaisu A. participate in study design and preparing the manuscript, Shafie A.A. supervised data analyses. All authors read and approved the final manuscript.

\section{Acknowledgements}

The authors would like to thank all physicians and endocrinologists at Hospital Pulau Pinang Endocrinology Clinic (especially Dr. Noor Azizah head of the department) and patients with diabetess who participated in and facilitated our work.

\section{References}

1. Haire-Joshu D, Glasgow RE, Tibbs TL. Smoking and Diabetes. Diabetes Care. 2004;27(1):s74-s5. 
2. International Diabetes Federation. IDF Diabetes Atlas Brussels, Belgium: International Diabetes Federation; 2019 [cited 2020 Auguest ]. 9th edition:[Available from: https://www.diabetesatlas.org.

3. Nilsson PM, Gudbjörnsdottir S, Eliasson B, Cederholm J. Smoking is associated with increased HbA1C values and microalbuminuria in patients with diabetes - data from the National Diabetes Register in Sweden. Diabetes Metab. 2004;30(3):261-8.

4. lino K, Iwase M, Tsutsu N, lida M. Smoking cessation and glycaemic control in type 2 diabetic patients. Diabetes Obes Metab. 2004;6(3):181-6.

5. Targher G, Alberiche M, Zenere MB, Bonadonna RC, Muggeo M, Bonora E. Cigarette smoking and insulin resistance in patients with noninsulin-dependent diabetes mellitus. J Clin Endocrinol Metab. 1997;82(11):3619-24.

6. Li WHC, Wang MP, Lam TH, Cheung YTY, Cheung DYT, Suen YN, et al. Brief intervention to promote smoking cessation and improve glycemic control in smokers with type 2 diabetes: a randomized controlled trial. Sci Rep. 2017;7:45902.

7. Pan A, Wang Y, Talaei M, Hu FB. Relation of smoking with total mortality and cardiovascular events among patients with diabetes: a meta-analysis and systematic review. Circulation. 2015;132(19):1795.

8. Śliwińska-Mossoń M, Milnerowicz $\mathrm{H}$. The impact of smoking on the development of diabetes and its complications. Diab Vasc Dis Res. 2017;14(4):265-76.

9. Radzeviciene L, Ostrauskas R. Smoking habits and the risk of type 2 diabetes: A case-control study. Diabetes Metab. 2009;35(3):192-7.

10. Willi C, Bodenmann P, Ghali WA, Faris PD, Cornuz J. Active smoking and the risk of type 2 diabetes. JAMA. 2007;298(22):2654-64.

11. Chaturvedi N, Stevens L, Fuller JH. Which features of smoking determine mortality risk in former cigarette smokers with diabetes? The World Health Organization Multinational Study Group. Diabetes Care. 1997;20(8):1266-72.

12. Eliasson B. Cigarette smoking and diabetes. Prog Cardiovasc Dis. 2003;45(5):405-13.

13. International Diabetes Federation. Global guideline for type 2 diabetes. Brussels: International Diabetes Federation; 2005.

14. Canga N, De Irala J, Vara E, Duaso MJ, Ferrer A, Martinez-Gonzalez MA. Intervention study for smoking cessation in diabetic patients: a randomized controlled trial in both clinical and primary care settings. Diabetes Care. 2000;23(10):1455-60.

15. Kottke TE, Solberg LI, Brekke ML, Conn SA. A controlled trial to integrate smoking cessation advice into primary care practice: Doctors Helping Smokers, Round III. J Fam Pract 1992;34:701-8.

16. Scemama O, Hamo-Tchatchouang E, Le Faou AL, Altman JJ. Difficulties of smoking cessation in diabetic inpatients benefiting from a systematic consultation to help them to give up smoking. Diabetes Metab. 2006;32(5):435 - 41.

17. Hokanson JM, Anderson RL, Hennrikus DJ, Lando HA, Kendall DM. Integrated tobacco cessation counseling in a diabetes self-management training program. Diabetes Educ. 2006;32(4):562-70. 
18. Ng N, Nichter M, Retna Siwi P, Prabandari YS, Muramoto M, Nichter M. Bringing smoking cessation to diabetes clinics in Indonesia. Chronic IIIn. 2010;6(2):125-35.

19. Welch G, Zagarins SE, Feinberg RG, Garb JL. Motivational interviewing delivered by diabetes educators: Does it improve blood glucose control among poorly controlled type 2 diabetes patients? Diabetes Res Clin Pract. 2011;91(1):54-60.

20. Tonstad S. Cigarette smoking, smoking cessation, and diabetes. Diabetes Res Clin Pract. 2009;85(1):413.

21. Tien CY, Tu S-T. To increase smoking cessation rate among diabetic smokers who participate in smoking cessation clinics by means of effective health education. Diabetes Res Clin Pract. 2016;120:S146.

22. El Sony A, Slama K, Salieh M, Elhaj H, Adam K, Hassan A, et al. Feasibility of brief tobacco cessation advice for tuberculosis patients: a study from Sudan. The International Journal of Tuberculosis and Lung Disease. 2007;11(2):150-5.

23. Slama K, Chiang CY, Enarson DA. Tobacco cessation and brief advice [Educational Series: tobacco and tuberculosis. Serialised guide. Tobacco cessation interventions for tuberculosis patients. Number 4 in the series]. The International Journal of Tuberculosis and Lung Disease. 2007;11(6):612-6.

24. Lin H, Lin Y, Zheng Y, Liu Z, Chang C. Design, development and randomised controlled trial of a smartphone application, 'QinTB', for smoking cessation in tuberculosis patients: study protocol. BMJ Open. 2019;9(12):e031204.

25. Campbell IA, Chaudhary RD, Holdsworth GMC, Lyne OD. Brief advice to tuberculosis patients in Nepal to stop smoking: a pilot study by the Britain Nepal Medical Trust. The International Journal of Tuberculosis and Lung Disease. 2014;18(12):1438-42.

26. Chen J, Chen Y, Chen P, Liu Z, Luo H, Cai S. Effectiveness of individual counseling for smoking cessation in smokers with chronic obstructive pulmonary disease and asymptomatic smokers. Exp Ther Med. 2014;7(3):716-20.

27. Lancaster T, Stead LF. Individual behavioural counselling for smoking cessation. The Cochrane database of systematic reviews [Internet]. 2017 2017/03//; 3:[CD001292 p.]. Available from: http://europepmc.org/abstract/MED/28361496 https://doi.org/10.1002/14651858.CD001292.pub3 https://europepmc.org/articles/PMC6464359 https://europepmc.org/articles/PMC6464359?pdf=render.

28. Y.H. C. Randomised controlled trials (RCTs)--sample size: the magic number? Singapore Med J. 2003;44:172-4.

29. Fiore MC, Jaen C, Baker T. Treating tobacco use and dependence: 2008 update. Rockville, MD2008.

30. Aziah M. Clinical practice guidelines on treatment of tobacco use and dependence 2003 [cited 2011 26-May]. Available from: http://www.treatobacco.net/en/uploads/documents/Treatment\%20Guidelines/Malaysia\%202003.pdf.

31. Bedfont. Helping People To Stop Smoking with a Smokerlyzer® CO Monitor 2010 [cited 2012 11-dec.]. Available from: http://www.bedfont.com/smokerlyzer. 
32. Leech NL, Barrett KC, Morgan GA. SPSS for Intermediate Statistics: Use and Interpretation. second ed. London: Lawrence Erlbaum Associates; 2005.

33. Fausset $C B$, Rogers WA, Fisk AD. Univariate and multivariate analysis of variance: A primer. Atlanta, GA: Georgia Institute of Technology School of Psychology - Human Factors and Aging Laboratory 2009.

34. Ministry of Health Malaysia, Persatuan Diabetes Malaysia (PDM), Academy of Medicine. Clinical practice guidelines, management of type 2 diabetes mellitus 2004 [cited 2012 10-dec.]. Available from: http://www.mems.my/file_dir/6684395974a1a58dae2698.pdf.

35. Kottke TE, Brekke ML, Solberg LI, Hughes JR. A randomized trial to increase smoking intervention by physicians: Doctors helping smokers, round I. JAMA. 1989;261(14):2101-6.

36. lino K, Iwase M, Tsutsu N, lida M. Smoking cessation and glycaemic control in type 2 diabetic patients. Diabetes Obes Metab. 2004;6(3):181-6.

37. Omvik P. How smoking affects blood pressure. Blood Press. 1996;5(2):71-7.

38. Lee D-H, Ha M-H, Kim J-R, Jacobs DR. Effects of Smoking Cessation on Changes in Blood Pressure and Incidence of Hypertension. Hypertension. 2001;37(2):194-8.

39. McEwen A, West R. Can stopping smoking cause hypertension? Nicotine Tobacco Res. 2002;4(3):34950.

40. Gepner AD, Piper ME, Johnson HM, Fiore MC, Baker TB, Stein JH. Effects of smoking and smoking cessation on lipids and lipoproteins: outcomes from a randomized clinical trial. Am Heart J. 2011;161(1):145-51.

\section{Figures}




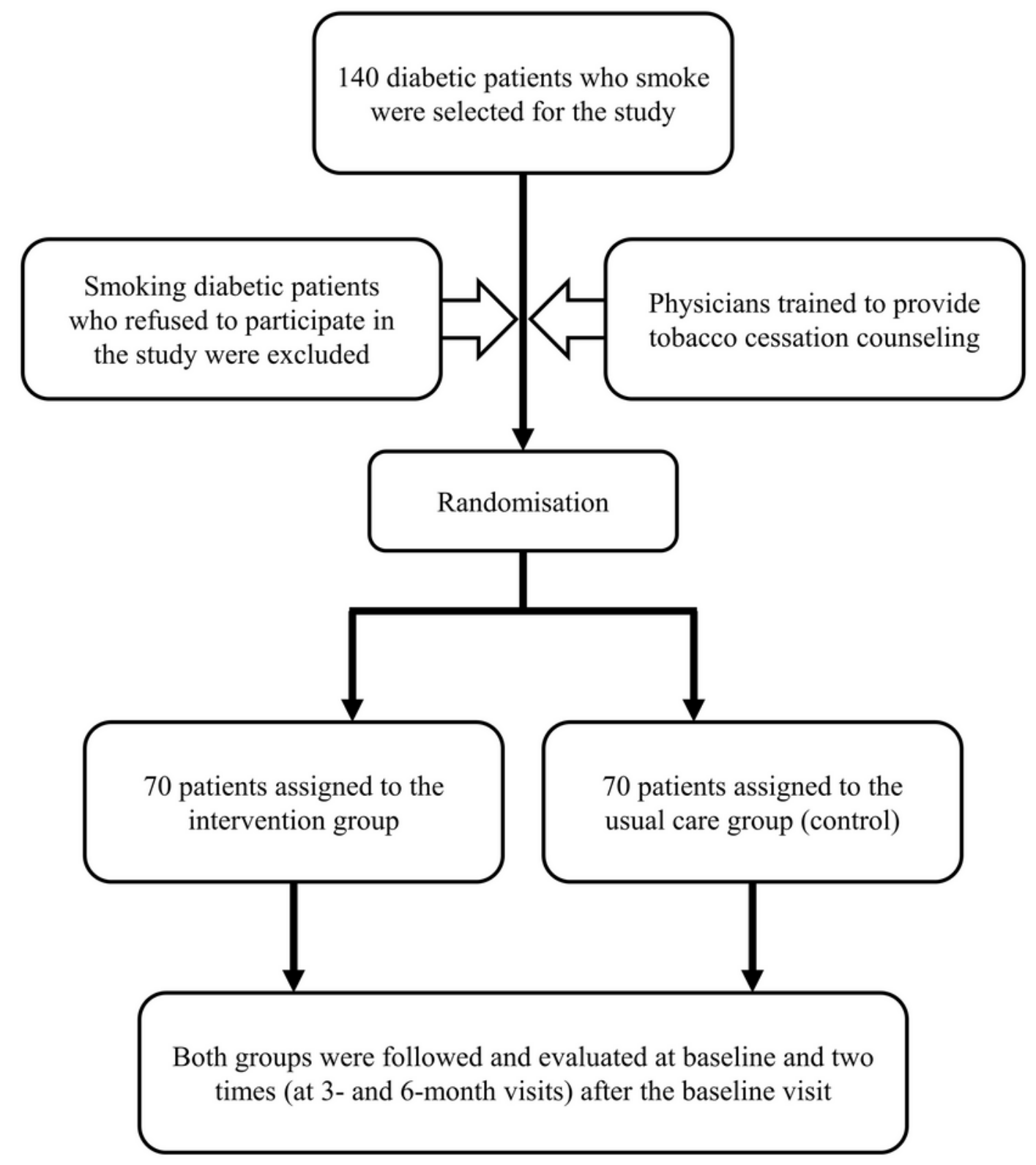

Figure 1

Study design

\section{Supplementary Files}

This is a list of supplementary files associated with this preprint. Click to download. 
- finalsupplementarrytables.docx 